\title{
PERMUTATION POLYNOMIALS OVER GALOIS FIELDS OF CHARACTERISTIC
}

\author{
${ }^{1}$ Dahiru, Z.L. and ${ }^{2}$ Lawan, A.M. \\ ${ }^{1}$ Department of Sciences, School of continuing Education, Bayero university, kano. \\ ${ }^{2}$ Department of Mathematical Sciences, Bayero University, Kano.
} Correspondence author: amlawan.mth@buk.edu.ng

\begin{abstract}
In this paper, a class of permutation polynomial known as o-polynomial over Galois fields of characteristic 2 was studied. A necessary and sufficients condition for a monomial $x^{2^{k}}$ to be an o-polynomial over $F_{2^{t}}$ is given and two results obtained by Gupta and Sharma (2016) were deduced.
\end{abstract}

\section{INTRODUCTION}

Let $q$ be a power of a prime $p$, and $F_{q}$ be the Galois field with $q$ elements. A polynomial $f \in F_{q}[X]$ is called a permutation polynomial (PP) if the associated polynomial function $f: x \rightarrow f(x)$ is a bijection of $F_{q}$. Permutation polynomials have been a subject of study and have many applications in combinatorics, coding theory, cryptography, design theory and so on.For basic concepts in permutation polynomials, see for example (G. L. Mullen and D. Panario, 2013; R. Lidl and H. Niederreiter, 1997). In general, permutation polynomials of finite fields are easy to construct without much consideration (there are $q$ ! permutation polynomials of $F_{q}$, all are given by the Lagranges interpolation), thus, the concern is on permutation polynomials with either simple or nice algebraic appearance or posses additional properties, such additional properties are require by applications of PPs in other areas of mathematics and engineering. In recent years, there have been significant progress in finding new classes of permutation polynomials. Let $F_{q}$ be a Galois field of order $2^{t}$ where $t \geq 1$. A permutation polynomial $f(x)$ over $F_{2}$ with $f(0)=0$ and $f(1)=1$, such that for each $v \in F_{2^{t}}$, the polynomial $f v(x)=\frac{f(x+v)+f(v)}{x}$ satisfying $f_{v}(0)=0$ is called an o-polynomial. (Lidl and Gary,1993), discussed some open problems on permutation polynomials (problem [P16] asked to determine all o-polynomials up to degree 6), (Gupta and Sharma, 2016) determined all o- polynomials up to degree 8 using the classification given by (L. E. Dickson,
1897).In this paper, using a technique known as multiplicative equivalent of permutation polynomils, we give necessary and sufficient condition for a monomial $x^{2^{k}}$ to be an opolynomial over $F_{2^{t}}$ and two results obtained by (Gupta and Sharma, 2016) were deduced.

\section{Preliminaries}

In this section we give definition and some relevant results needed for the understanding of this paper.

Definition 2.1: Two permutation polynomials $f(x)$ and $g(x)$ in $F_{q}[\mathrm{x}]$ are said to be multiplicative equivalent if there exist an integer $1 \leq d \leq q-1$ such that $\operatorname{gcd}(d, q-1)=1$ and $f(x)=g(x)^{d}$. Moreover, $f(x)$ and $g(x)$ are equivalent if $f(x)=c g(a x+b)+d$ where $d, b \in F_{q}$ and $a, c \in F_{q}{ }^{*}$.

A monomial is a polynomial consisting of only one term. Any polynomial of degree one is called linear polynomial.

Lemma 2.2 (Lidl and Niederreiter, 1997; Lemma 7.1): Let $f(x)$ be a polynomial over $F_{q}[X]$, then the following definitions are equivalent:

(i) $\quad f(x)$ is a permutation polynomial on $F_{q}$.

(ii) The function $f: x \rightarrow f(x)$ is one to one for each $x \in F_{q}$.

(iii) The function $f: x \rightarrow f(x)$ is onto $x \in$ $F_{q}$.

(iv) $\quad f(x)=a$ has a solution in $F_{q}$ for each $a \in F_{q}$.

(v) $\quad f(x)=a$ has a unique solution in $F_{q}$ for each $a \in F_{q}$. 
BAJOPAS Volume 13 Number 2, December, 2020

Theorem 2.3 (J. Li. Chandler and Xiang, 2010; Theorem 3.4). There are no permutation polynomials of degree 6 over $F_{2}$, when $t>4$ is even.

Theorem 2.4 (Lidl and Niederreiter, 1998; Theorem 7.8)

(i) Every linear polynomial over $F_{q}$ is a permutation polynomial of $F_{q}$

(ii) The monomial $x^{i}$ is a permutation polynomial if and only if $\operatorname{gcd}(i, q-1)=1$.

\section{Some results on o-polynomials}

In this section, we present some important results on o-polynomials which are needed to achieve our objectives.

Theorem 3.1(Gupta and Sharma, 2016; Theorem 2.9 ). The coefficient of each term of odd power in an o-polynomials is zero.

As a consequence, we deduce the following corollary.

Corollary 3.2: There are no o-polynomials of odd degree.

Proof. Follows from the definition of o-polynomialand from the fact that if the coefficient of odd power is zero, then the whole term will be zero and the rest of the results follows directly from Theorem 3.1.

Table 1 below is the complete list of all o-polynomials of degree 2,4,6 and 8 obtained by (Gupta and Sharma, 2016).

\begin{tabular}{|c|c|}
\hline Degree & Polynomial \\
\hline 2 & $f(x)=x^{2}$ \\
\hline 4 & $f(x)=x^{4}$ \\
\hline 6 & $f(x)=x^{6}, a_{1} x^{6}+a_{2} x^{4}+a_{1} x^{2}, a_{3} \neq 0, a_{1}+a_{2}+a_{3}=1, a_{1} a_{3}=a_{2}$ for odd $t$ \\
\hline 8 & $f(x)=x^{8}, t \equiv 0 \bmod 3$ \\
\hline
\end{tabular}

\section{Main Results}

In this section we present the necessary and sufficient condition for a monomial $x^{2^{k}}$ to be an opolynomial over $F_{2^{t}}$ and two results obtained by (Gupta and Sharma, 2016) were deduced.

Theorem 4.1:A monomial $x^{2^{k}}$ is an o-polynomial over $F_{2^{t}}$ if and only if $\operatorname{gcd}(k, t)=1$, for $k \in \mathbb{N}$. Proof: Suppose $x^{2^{k}}$ is an o-polynomial over $F_{2^{t}}$, then $\operatorname{gcd}\left({ }^{\prime} 2^{\mathrm{k}}, 2^{\mathrm{t}}-1\right)=1$. Notice that, since $x^{2^{k}}$ is a permutation over $F_{2^{t}}$ satisfying $f(0)=0, f(1)=1$, and for each $v \in F_{2^{t}}$ we have $\frac{x^{2^{k}}+v^{2^{k}}+v^{2^{k}}}{x}=\frac{x^{2^{k}}}{x}$ $=x^{2^{k}} \cdot x^{-1}=x^{2^{k}-1}$ which is also a permutation polynomial. This implies that $\operatorname{gcd}\left(2^{k}-1,2^{t}-1\right)=1$, thusgcd $(k, t)=1$.

Conversely, suppose $\operatorname{gcd}(k, t)=1$, this impliesgcd $\left(2^{k}-1,2^{t}-1\right)=1$. We may without loss of generality, consider $f(x)=x^{2^{k}-1}$. By (theorem $2.4(\mathrm{ii})$ ), $x^{2^{k}-1}$ is a permutation polynomial over $F_{2} t$ and by direct computation we have $f(x)=x^{2^{k}}$ as a monomialo-polynomials over $F_{2}$.

Next, we support theorem 4.1 with the following example.

Example 4.2: Let $\mathrm{k}=4$ and consider the polynomial $f(x)=x^{2^{4}}=x^{16}$. To prove the existence of this o-polynomial, we proceed as follows. Since $\operatorname{gcd}\left(2^{4}, 2^{t}-1\right)=1$ for all $t \in \mathbb{N}$, then $f(x)=x^{2^{4}}$ is a permutation polynomial. Notice that $\operatorname{gcd}(4, t)=1$ whenever $t$ is odd and by (theorem 4.1), $f(x)=x^{2^{4}}=x^{16}$ is an o-polynomial.

As a consequence of the above theorem we deduced two results obtained by (Gupta and Sharma, 2016) as follows

Corollary 4.3:The polynomial $f(x)=x^{2}$ is a monomial o-polynomial of degree 2 .

Proof: There are 4polynomials of degree 2 over $F_{2} t$, these are $f_{1}(x)=x^{2}, f_{2}(x)=x^{2}+x, f_{3}(x)=$ $x^{2}+x+1$ and $f_{4}(x)=x^{2}+1$. Clearly for the polynomials $f_{2}(x), f_{3}(x)$ and $f_{4}(x)$, the polynomial function $f(x)=a$ has no unique solution for each $a \in F_{2}$, hence it is not a permutation polynomials by (lemma 2.2(iv)). For $f_{1}(x), \operatorname{gcd}\left(2,2^{t}-1\right)=1$ for all $t \in \mathbb{N}$, then by (lemma 2.4) $f_{1}(x)$ is a permutation polynomial. Moreover, since the $\operatorname{gcd}(1, t)=1$ for all positive number $t$ and by (theorem 4.1), $f_{1}(x)$ is an o-polynomial of degree 2 . Hence $f_{1}(x)=x^{2}$ is a monomial o-polynomial of degree 2 . Corollary 4. 4: The polynomial $f(x)=x^{4}$ is a monomial o-polynomial of degree 4 .

Proof. Here the only possible choices are polynomial of the form $(x)=a_{1} x^{4}+a_{2} x^{2}$, where $a_{1}, a_{2} \in F_{2^{t}}$. Let $f(x)=g\left(x^{2}\right)$ with $g(x)=a_{1} x^{2}+a_{2} x$, then $f(x)$ and $g(x)$ are multiplicative equivalent since there exists a positive integer $1 \leq 2 \leq 2^{t}-1$ with $\operatorname{gcd}\left(2,2^{t}-1\right)=1$. Since by definition $f(x)$ is a permutation polynomial if and only if $g(x)$ is a permutation polynomial. From (corollary 4.3$), g(x)$ 
BAJOPAS Volume 13 Number 2, December, 2020

is a permutation polynomial if $a_{2}=0, a_{1}=1$, and $g(x)=x^{2}$. Hence, $f(x)=x^{4}$ is a permutation polynomial. Notice that the $\operatorname{gcd}(4, t)=1$ whenever $t$ is a positive odd number and by (theorem 4.1), the polynomial $x^{4}$ is a monomial o-polynomial.

\section{CONCLUSION}

Permutation polynomials over Galois fields are both interesting and important in theory and in many applications. We have studied some properties of monomialso-polynomials over

\section{REFERENCES}

Dickson, L.E, (1897). The Analytic Representation of Substitutions on a Power of a Prime Number of Letters with a Discussion of the Linear Group, part I. Annals of Mathematics. 11:65-120.

Gupta,R., and Sharma, R.K.(2016).On Permutation Polynomials over Finite Fields of Characteristic Two. Journal of Algebra and its Applications.

Laigle-chapuy, Y. (2007). Permutation polynomials and Applications to Coding Theory. Journal of Finite Fields and its Applications

Li.J., Chandler, D.B. and Xiang. Q.(2010).Permutation polynomials of degree 6 or 7 over Finite fields of characteristic 2.Journal of Finite Fields and its Applications.
Galois fields of characteristic 2and gave a necessary and sufficient condition foron monomials polynomials to be an o-polynomials, some existing results were deduced from our results.

Lidl, R. and Gary, L.M. (1993). When Does a Polynomial over a Finite Field Permute the Elements of the Field?. Monthly Magazine of American Math.

Lidl, R.and Niederreiter, H. (1997). Finite Fields, $2^{\text {nd }}$ edition. Cambridge University, Press. Cambridge, United Kingdom.

Xiang-dong, Hou. (2015). Permutation Polynomials over Finite Fields A survey of recent advances. Finite fields and Applications.

X. Hou., (2011). Two classes of Permutation Polynomials over Finite Fields. J. Comb. Theory, series

A.T. Hellesth and V. Zinoviev, (2003). New Kloosterman sums identities over $F_{2} m$ for all $m$. Finite fields and Applications. 